\title{
Emerging role of selective autophagy in human diseases
}

\section{Kenji Mizumura, Augustine M. K. Choi and Stefan W. Ryter*}

Joan and Sanford I. Weill Department of Medicine, Weill Cornell Medical Center, New York-Presbyterian Hospital - Weill Cornell Medical College, New York, NY, USA

\section{Edited by: \\ Frank Wagener, Radboud University Nijmegen Medical Centre, \\ Netherlands}

\section{Reviewed by:}

Stephen Tait, University of Glasgow, UK

Danhui Liu, Wenzhou Medical

College, China

Frank Wagener, Radboud University Nijmegen Medical Centre,

Netherlands

\section{*Correspondence:}

Stefan W. Ryter, Joan and Sanford I. Weill Department of Medicine, Weill Cornell Medical Center,

New York-Presbyterian Hospital - Weill Cornell Medical College, 525 East 68th Street, Room M-522, P.O. Box 130, New York, NY 10065, USA e-mail: str2020@med.cornell.edu
Autophagy was originally described as a highly conserved system for the degradation of cytosol through a lysosome-dependent pathway. In response to starvation, autophagy degrades organelles and proteins to provide metabolites and energy for its pro-survival effects. Autophagy is recognized as playing a role in the pathogenesis of disease either directly or indirectly, through the regulation of vital processes such as programmed cell death, inflammation, and adaptive immune mechanisms. Recent studies have demonstrated that autophagy is not only a simple metabolite recycling system, but also has the ability to degrade specific cellular targets, such as mitochondria, cilia, and invading bacteria. In addition, selective autophagy has also been implicated in vesicle trafficking pathways, with potential roles in secretion and other intracellular transport processes. Selective autophagy has drawn the attention of researchers because of its potential importance in clinical diseases. Therapeutic strategies to target selective autophagy rather than general autophagy may maximize clinical benefit by enhancing selectivity. In this review, we outline the principle components of selective autophagy processes and their emerging importance in human disease, with an emphasis on pulmonary diseases.

\section{Keywords: autophagy, mitophagy, lung diseases, ciliophagy, xenophagy}

\section{INTRODUCTION}

Autophagy is a lysosomal degradation system by which the cell can recycle its cytoplasmic components (Mizushima and Komatsu, 2011). At present, three different types of autophagic pathways have been reported, named as macroautophagy, microautophagy, and chaperone-mediated autophagy (Mizushima and Komatsu, 2011). Of these, macroautophagy is the best-characterized and most well-known form, often referred to simply as "autophagy."

Autophagy proceeds through sequential steps, beginning with the generation of autophagosomes from an isolation membrane and followed by elongation to form a mature autophagosome which captures cytosolic cargo (Mizushima and Komatsu, 2011). Genetic studies in yeast have identified a series of autophagyrelated genes (ATGs) shown to be essential for the autophagy process (Tsukada and Ohsumi, 1993; Klionsky et al., 2003). Among these, microtubule associated protein 1 light chain-3 (LC3), a homologue of yeast Atg8, is converted from a cytosolic form (LC3-I) to its phosphatidylethanolamine-conjugated form (LC3-II) which targets to autophagic membranes (Mizushima et al., 2010). Autophagosome formation is also regulated by the autophagy protein Beclin 1 (homolog of yeast Atg6; Liang et al., 1999).

The membrane origin of autophagosomes remains unclear. Although the endoplasmic reticulum (ER), mitochondria and plasma membrane have been reported as the membrane source, recent studies have also suggested that the ER-mitochondria contact site is important in autophagosome formation (Hailey et al., 2010; Ravikumar et al., 2010; Tooze and Yoshimori, 2010; Hamasaki et al., 2013). Subsequently, the autophagosome containing the cytosolic components and organelles fuses with the lysosome to form the autolysosome where the sequestered cargo is degraded (Mizushima and Komatsu, 2011). Initial studies on the molecular mechanisms of autophagy have largely focused on the early stage, however, precise mechanisms of the late stage where the autophagosome fuses with the lysosome have also been revealed (Shen and Mizushima, 2014). Recent studies have demonstrated that the transcription factor EB (TFEB), a master gene for lysosomal biogenesis, coordinates the autophagic process by driving expression of autophagy and lysosomal related genes (Settembre et al., 2011). An autophagosomal soluble $N$-ethylmaleimidesensitive factor attachment protein receptor (SNARE) has been identified as the regulator of autophagosome-lysosome fusion (Itakura et al., 2012).

Once, autophagy was simply regarded as a non-specific degradation system, however, recent research shows that autophagy can selectively degrade specific targets in processes referred to as "selective autophagy" (Levine et al., 2011; Youle and Narendra, 2011). Each selective autophagy subtype was named after its specific targets, for example: aggregated proteins (aggrephagy; Yamamoto and Simonsen, 2011), mitochondria (mitophagy; Youle and Narendra, 2011), pathogens (xenophagy; Levine et al., 2011), and cilia (ciliophagy; Cloonan et al., 2014). Selective autophagy is also related to vesicle trafficking pathways, and its importance in secretion and other intracellular transport processes is rapidly increasing (Stolz et al., 2014).

Previous studies suggest that autophagy is relevant to human diseases, including pulmonary diseases (Levine and Kroemer, 2008; Choi etal., 2013). Furthermore, convincing evidence that selective autophagy may be implicated in human disease 
has been reported (Gomes and Dikic, 2014; Redmann et al., 2014). This led us to the hypothesis that selective autophagy would impact the pathogenesis of pulmonary diseases. In this review, we will examine the considerable evidence emerging for the role of selective autophagy in the pathogenesis of complex pulmonary diseases. A better understanding of the role(s) of selective autophagy in disease pathogenesis may help design more specific therapies for the treatment of pulmonary diseases, and other diseases where autophagy may contribute to pathogenesis.

\section{SELECTIVE AUTOPHAGY}

Selective autophagy can deliver a wide range of cargo to the lysosome, including protein aggregates, whole organelles (e.g., mitochondria), and intracellular pathogens (Stolz et al., 2014). Although the mechanisms of selective degradation remain incompletely understood, several reports suggest that ubiquitination of substrates may serve as general tag for selective autophagy in mammalian cells (Kirkin et al., 2009). Recent studies have described important functions of Atg8 family proteins in selective autophagy, including interactions with cargo receptors and components of the basal autophagy machinery, and in the regulation of autophagosome biogenesis (Kaufmann etal., 2014; Nath etal., 2014; Sawa-Makarska et al., 2014). To evaluate the inclusive list of selective autophagy processes currently in the literature is beyond the scope of this review; we will therefore focus on the three types of selective autophagy most related to pulmonary diseases; mitophagy, xenophagy, and ciliophagy.

\section{MITOPHAGY}

Mitophagy is a selective mechanism for the elimination of mitochondria through the autophagic machinery (Youle and Narendra, 2011). Two major mitophagy-related proteins, Parkin and PTENinduced putative kinase protein 1 (PINK1), have been linked to the pathogenesis of Parkinson's disease (Youle and Narendra, 2011). A proposal for the mechanism of mitophagy is that damaged and depolarized mitochondria stabilize PINK1 which in turn recruits the E3 ubiquitin ligase, Parkin. Parkin then ubiquitinylates various mitochondrial outer membrane proteins including mitofusins MFN1, MFN2 (Gegg et al., 2010), voltage dependent anion channel (VDAC; Geisler et al., 2010) and mitochondrial rho GTPase (MIRO; Wang et al., 2011); and induces mitophagy by the recruitment of autophagy receptors such as p62 (Geisler et al., 2010). However, several previous reports are suggestive of PINK1-dependent, but Parkin-independent, mitophagy. Gp78 E3 ubiquitin ligase overexpression causes mitophagy that is independent of Parkin (Fu et al., 2013). Mice genetically deficient in Pink1 were resistant to Staphylococcus aureus-induced acute lung injury (ALI). PINK1 was found to interact with an alternative ubiquitin E3 ligase component, F-box only protein 15 (Fbxo15), which promoted mitochondrial instability in this model (Chen et al., 2014a). Furthermore, although mitophagy was generally considered to serve as an intrinsic mitochondrial quality control system, it has been reported that mitophagy may trigger cell death (Sentelle et al., 2012; Mizumura et al., 2014). Now, mitophagy is generally recognized as a potential modulator of the pathogenesis of disease with either protective or harmful consequences.

\section{XENOPHAGY}

Autophagy can contribute to the immune response by providing a mechanism for the selective intracellular degradation of invading pathogens, a process termed "xenophagy." Invading bacteria are tagged for removal with ubiquitin. Autophagy receptors including p62, nuclear domain 10 protein 52 (NDP52) and optineurin recognize ubiquitinated pathogens and target them to autophagosomes (Gomes and Dikic, 2014). Besides its direct role in pathogen clearance, xenophagy may also serve host defenses by enhancing immune recognition of infected cells via the generation of antigenic bacterial peptides (Yano and Kurata, 2011). Meanwhile, some bacteria, such as S. aureus and Anaplasma phagocytophilum, can use the host autophagosomes for replication (Schnaith et al., 2007; Niu et al., 2008). These bacteria can not only block autophagosomal maturation and acidification, but also can proliferate in LC3-positive compartments (Gomes and Dikic, 2014).

\section{CILIOPHAGY}

Recently, we have demonstrated that an autophagy-dependent pathway regulates cilia length (Lam et al., 2013), a process named "ciliophagy." We have shown that the cytosolic deacetylase histone deacetylase 6 (HDAC6) mediates ciliophagy. Pampliega et al. (2013) also reported that autophagy negatively regulate ciliogenesis by degrading intraflagellar transport protein 20 homolog (IFT20). On the other hand, it has reported that autophagy removes oral-facial-digital syndrome 1 protein (OFD1) from centriolar satellites to promote ciliogenesis (Tang et al., 2013). However, the precise mechanisms by which autophagy can regulate these conflicting processes remains to be elucidated (Wrighton, 2013).

\section{SELECTIVE AUTOPHAGY IN COPD}

Chronic obstructive pulmonary disease (COPD) contributes significantly to the global burden of disease as the fourth leading cause of mortality worldwide, however, the pathogenesis of this disease remains incompletely understood (Dal-Re, 2011; Vestbo et al., 2013). We previously reported increased autophagosome numbers and increased expression of LC3B-II, the active form of $\mathrm{LC} 3 \mathrm{~B}$, in human lung specimens from patients with COPD (Chen etal., 2010). In an in vivo emphysema model, genetic deletion of specific autophagy proteins reduced airspace enlargement (Chen et al., 2010). More recently, we demonstrated that mitophagy regulates necroptosis, a form of programmed necrosis, which contributes to the pathogenesis of COPD (Mizumura et al., 2014). Cigarette smoke (CS) exposure induced mitophagy through the stabilization of the mitophagy regulator PINK1 in pulmonary epithelial cells. Mice genetically deficient in PINK1 were protected against mitochondrial dysfunction, airspace enlargement, and mucociliary clearance (MCC) disruption during CS exposure. The mitochondrial division/mitophagy inhibitor Mdivi-1 protected against CS-induced cell death and mitochondrial dysfunction, and reduced the phosphorylation of mixed lineage kinase domain-like protein (MLKL), a substrate for receptor-interacting serine/threonine-protein kinase 
3 (RIP3) in the necroptosis pathway. In this study, we have shown that significant mitochondrial depolarization occurred in pulmonary epithelial cells in response to CS extract (CSE) exposure. Moreover, our results suggest that active mitophagy may alter mitochondrial membrane integrity, and lead to the induction of necroptosis. However, the precise mechanism by which mitophagy can serve to aggravate mitochondrial injury in the CS exposure model remains obscure. One possible hypothesis is that CS-induced aberrant mitophagy may cause an increase in the population of impaired mitochondria (Figure 1A). In addition, as dose-response effects of autophagy/mitophagy have been proposed, we cannot completely exclude the possibility that mitophagy may also contribute to mitochondrial quality control for its pro-survival role during mild CS exposure (Frank et al., 2012; Sureshbabu and Bhandari, 2013; Schiavi and Ventura, 2014). Further studies are necessary to improve the understanding of the role of mitophagy in the pathogenesis of COPD.

We also reported that ciliophagy, the consumption of cilia components by autophagy, regulates cilia length during CS exposure (Figure 1B; Lam etal., 2013; Cloonan etal., 2014). Impaired airway clearance caused by cilia shortening prevents the elimination of pathogens from the airways and may cause recurrent respiratory infections that exacerbate COPD. We demonstrated that autophagy-impaired $\left(B e c n 1^{+/-}\right.$or $\left.M a p 1 l c 3 B^{-/-}\right)$mice, as well as tracheal epithelial cells isolated from these mice, resisted CS-induced cilia shortening. We identified the cytosolic deacetylase HDAC6 as a critical regulator of autophagy-mediated cilia shortening during CS exposure (Lam etal., 2013; Cloonan etal., 2014).

In contrast, previous studies have demonstrated defective autophagy in CS-exposed macrophages (Monick et al., 2010). Such a deficit in autophagy/xenophagy was observed in the alveolar macrophages of smokers and was proposed to lead to recurrent infections in smokers, since CS exposure impairs delivery of bacteria to the lysosomes.

\section{SELECTIVE AUTOPHAGY IN RESPIRATORY INFECTION AND SEPSIS}

The mechanism of antibacterial autophagy in Mycobacterium tuberculosis (Mtb) infection is well-characterized. The lungs are the major site for Mtb infection. Mtb employs a unique strategy for survival that interferes with the fusion between phagosomal compartments containing Mtb and lysosomes (Vergne et al., 2004). Despite the availability of anti-TB drugs, recent reports have identified cases of totally drug-resistant TB (Loewenberg, 2012; Udwadia etal., 2012). Since new therapeutic agents that have different mechanisms of action from conventional anti-TB drugs are needed to prevent the development of drug resistance, bacterial autophagy (xenophagy) has drawn attention as a candidate therapeutic target. Previous studies have demonstrated that polymorphisms in the immunity-related GTPase family $M$ protein (IRGM) gene are linked to increased susceptibility to Mtb infection, and that IFN- $\gamma$ induced IRGM regulates autophagy to eliminate mycobacteria in human macrophages (Figure 2A; Singh et al., 2006; Intemann et al., 2009). Recent studies have revealed that Mtb extracellular DNA activates ubiquitin-mediated selective autophagy through phagosomal permeabilization (Figure 2B; Watson et al., 2012). The bacterial early secretory antigenic target 6 (ESAT-6) system 1 (ESX-1) secretion system mediates phagosomal permeabilization to enable the ubiquitin-mediated autophagy pathway access to phagosomal Mtb. The stimulator of interferon genes (STING)-dependent cytosolic pathway recognizes extracellular bacterial DNA and tags bacteria with ubiquitin. Autophagy receptors, p62, and NDP52, recognize ubiquitinated $\mathrm{Mtb}$ and target them to autophagosomes. Several therapies that involve enhancing autophagy activity also have been proposed to be effective against Mtb infection. The antiprotozoal drug nitazoxanide and its active metabolite tizoxanide strongly stimulate autophagy through inhibition of mTORC1 signaling, which in turn prevents intracellular proliferation of Mtb (Lam et al., 2012). Vitamin D has revealed therapeutic benefits in persons with HIV and Mtb infection through the activation of autophagy (Campbell and Spector, 2012).

Autophagy has been implicated in the regulation of inflammation, particularly the regulation of the inflammasome pathway. Inflammasomes represent an inflammatory signaling platform activated by infection or stress that regulate the maturation and secretion of pro-inflammatory cytokines (e.g., IL-1 $\beta$ and IL-18; Schroder and Tschopp, 2010). Along with Zhou et al, we have demonstrated that suppression of autophagy causes the accumulation of damaged ROS-producing mitochondria, whereas activates the NLRP3 inflammasome (Figure 2C; Nakahira et al., 2011; Zhou etal., 2011). In this study, we also found that the NLRP3 inflammasome and mitochondrial ROS production regulate cytosolic translocation of mitochondrial DNA (mtDNA) in macrophages, which in turn contributed to the secretion of IL-1 $\beta$ and IL-18. Importantly, these findings are consistent with the observation that IL- $1 \beta$ and IL-18 are increased in patients with sepsis in the medical intensive care unit (ICU; Nakahira et al., 2011; Dolinay et al., 2012). Moreover, we demonstrated that increased mtDNA levels in plasma are associated with ICU mortality, and inclusion of mtDNA level improves risk prediction in medical ICU patients (Nakahira et al., 2013). Recently, we have also demonstrated that carbon monoxide (CO) confers protection in sepsis by enhancing Beclin 1-dependent autophagy and phagocytosis (Lee et al., 2014). CO enhanced bacterial phagocytosis in $B e c n 1^{+/+}$but not $B e c n 1^{+/-}$mice in vivo and in corresponding cultured macrophages, which indicates that $\mathrm{CO}$ may induce xenophagy. These results suggest that $\mathrm{CO}$ gas may represent a novel therapy for patients with sepsis.

\section{SELECTIVE AUTOPHAGY IN HYPEROXIA AND ACUTE LUNG INJURY}

We have demonstrated that autophagy is implicated in the pathogenesis of ALI (Tanaka etal., 2012). Although mechanical ventilation with high concentrations of oxygen is required to manage patients with severe respiratory failure, prolonged exposure to hyperoxia can result in lung injury. Hyperoxia can induce autophagy activity. Depletion of LC3B by RNA interference reduced cell viability under hyperoxic conditions (Tanaka 


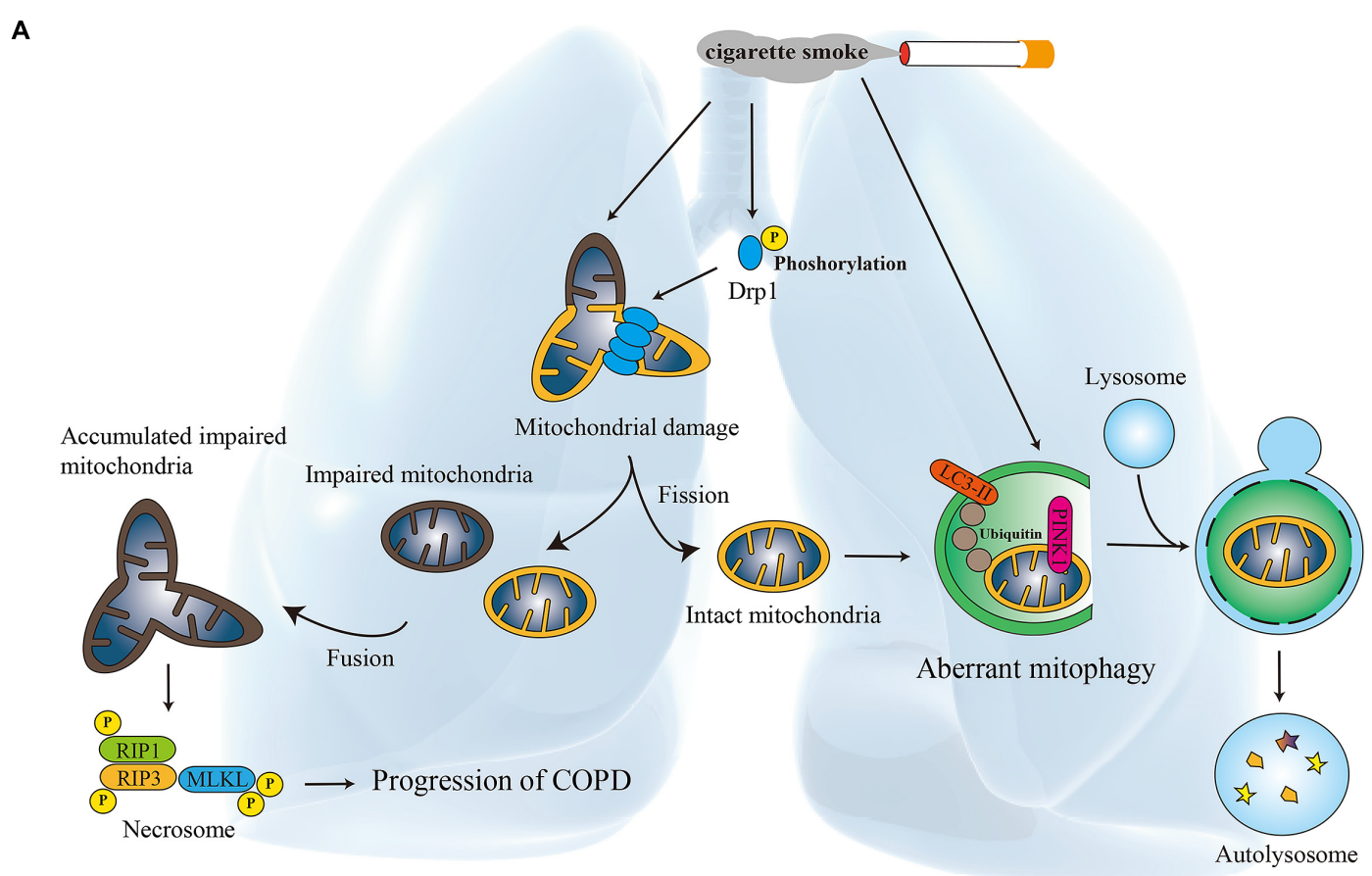

B

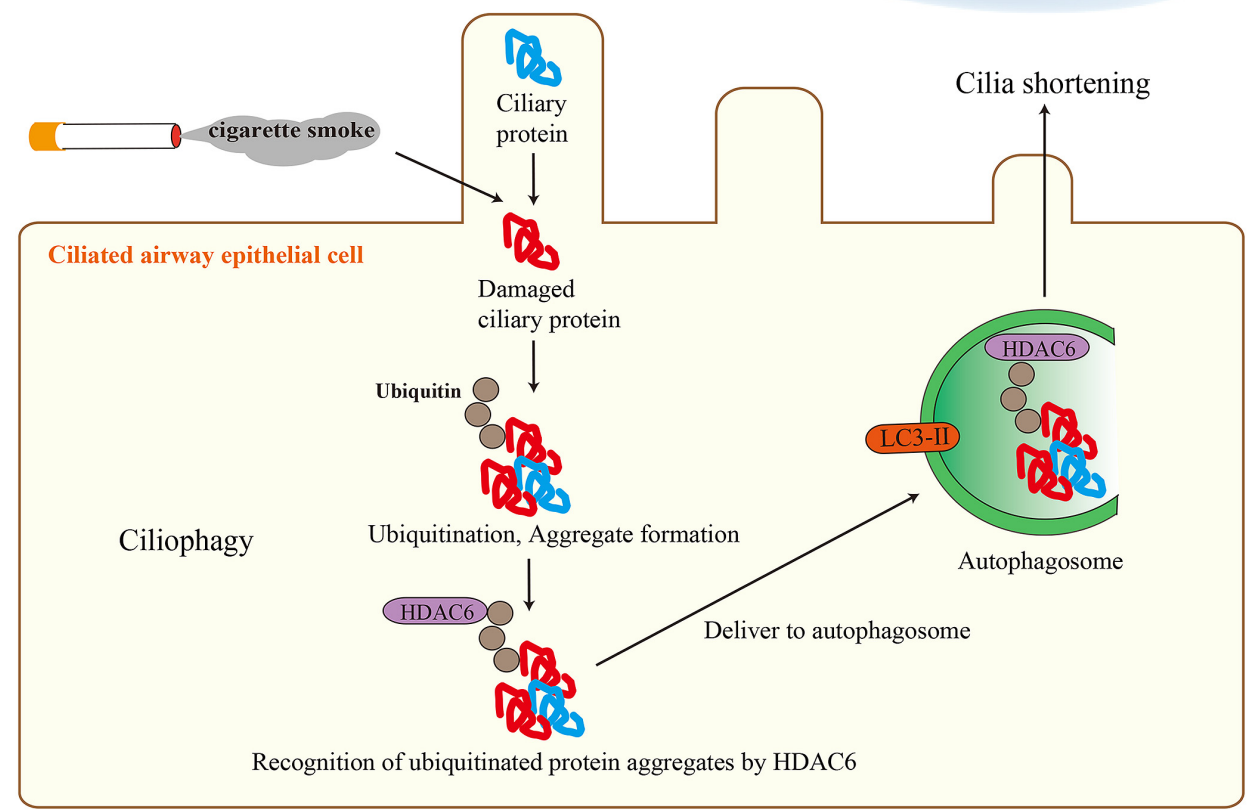

FIGURE 1 | Selective autophagy in chronic obstructive pulmonary disease (COPD). (A) The role of mitophagy in COPD. Cigarette smoke (CS) induced mitochondrial fission and PINK1-dependent mitophagy in epithelial cells independently from mitochondrial damage. This aberrant mitophagy may cause the increase in the population of impaired mitochondria, which leads to the initiation of necroptosis. (B) The role of ciliophagy in COPD. CS induces oxidative stress, which causes cilia protein damage. Damaged cilia proteins are ubiquitinated which promotes aggregate formation. HDAC6 recognizes ubiquitinated protein aggregates and delivers them to autophagosomes. This degradation of cilia proteins, through an autophagy-dependent process termed "ciliophagy," was associated with cilia shortening. et al., 2012). We also investigated the molecular mechanism by which autophagy can confer cytoprotection in lung epithelial cells after hyperoxia (Liang etal., 2013). Cellular homeostasis requires the constant formation of the $\mathrm{p} 62 / \mathrm{LC} 3 \mathrm{~B} /$ truncated $\mathrm{BH} 3$-interacting domain death agonist (tBID) complex under normal conditions, however, hyperoxia leads to dissociation of the p62/LC3B/tBID complex, which stops the translocation of tBID into lysosome for degradation. Increased tBID causes cytochrome $\mathrm{c}$ release from the mitochondria and subsequent caspase-dependent cell death. These results suggest that the autophagy may have protective function during the pathogenesis of ALI, especially under hyperoxia. 


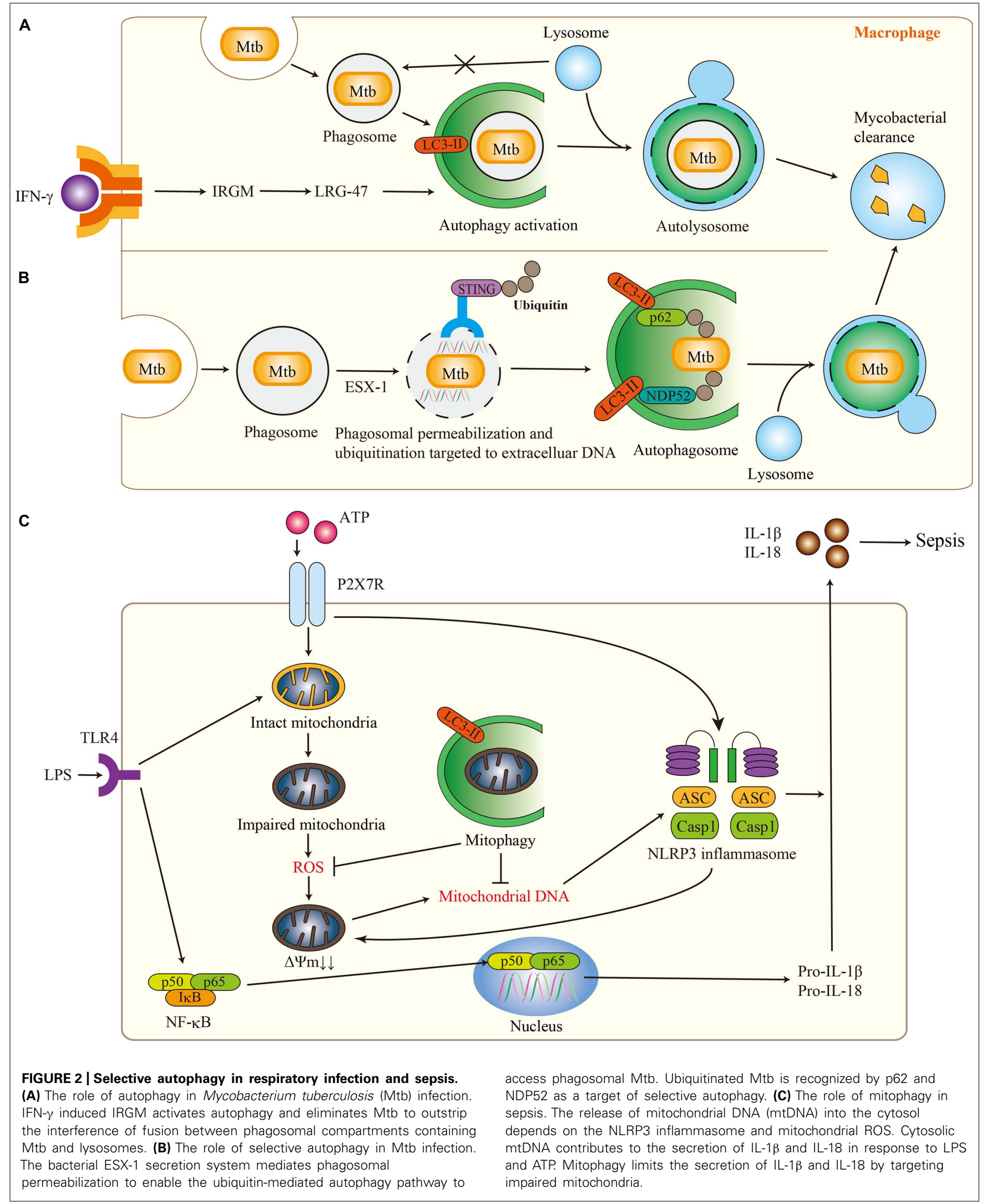


It has been reported that mechanical ventilation and hyperoxia cause pulmonary mitochondrial dysfunction (Ratner et al., 2009, 2013; Waxman and Kolliputi, 2009). Given that mitochondrial damage can induce mitophagy, it is reasonable to presume that mitophagy may play a role in the pathogenesis of ALI (Youle and Narendra, 2011). Indeed, the role of mitophagy in hyperoxia has been reported. Genetic deletion of PINK1 or PINK1 silencing in the lung endothelium increased susceptibility to hyperoxia via alterations in autophagy/mitophagy (Zhang etal., 2014). NLRP3 may regulate autophagy/mitophagy via PINK1 during hyperoxia. Consistent with a role for autophagy, these results also suggest that mitophagy may have protective function during the pathogenesis of ALI.

\section{SELECTIVE AUTOPHAGY IN THE HYPOXIA RESPONSE AND PULMONARY HYPERTENSION}

Hypoxia results in secondary pulmonary hypertension (PH). Hypoxic $\mathrm{PH}$ is a progressive and often fatal complication of chronic lung disease (Semenza, 2011). Chronic hypoxia induces pulmonary arterial vascular smooth muscle (PAVSM) cell proliferation, which is a major cause of PH (Stenmark et al., 2006). We previously demonstrated that elevated occurrences of autophagy have been observed in lung tissue from patients with PH. Mice genetically deficient in LC3B demonstrated increased indices of PH after chronic hypoxia (Lee et al., 2011). Chloroquine, the inhibitor of autophagy, has been reported to prevent progression of experimental PH (Long et al., 2013). Furthermore, as the inhibition of mTOR complex 1 (mTORC1) has been shown to induce autophagy, previous studies demonstrated that blockade of mTORC1 has anti-proliferative effects on pulmonary vascular cells (Krymskaya et al., 2011; Wang et al., 2014). These results suggest that autophagy may have a protective function during the pathogenesis of PH. LC3 and mTOR pathway are attracting attention as potential therapeutic targets in hypoxia-induced PH (Lahm and Petrache, 2012; Goncharova, 2013).

The mitochondrial outer-membrane protein FUN14 domaincontaining protein 1 (FUNDC1) mediates hypoxia-induced mitophagy in mammalian cells (Liu etal., 2012). FUNDC1 interacted with LC3; and knockdown of endogenous FUNDC1 significantly prevented hypoxia-induced mitophagy, which could be reversed by the expression of wild-type FUNDC1. Hypoxia can dephosphorylate FUNDC1 at serine 13 through serine/threonineprotein phosphatase PGAM5 for the induction of mitophagy (Chen et al., 2014b; Wu et al., 2014). These results suggest that mitophagy may contribute to mitochondrial quality control in hypoxia. However, hypoxia-induced mitophagy has also been reported to cause apoptosis in cardiomyocytes (Yan et al., 2014). The role of mitophagy in the pathogenesis of $\mathrm{PH}$ remained incompletely understood.

\section{CONCLUSION}

Although autophagy originally was considered as a simple bulk degradation system for cellular components, accumulating evidence demonstrates that autophagy can selectively degrade specific targets. Selective autophagy plays a complex role in human diseases where it can have both protective and injurious effects. However, when viewed in the light of evidence that many cellular functions can have both protective and injurious effects, it is likely that selective autophagy may also act as a double-edged-sword in the pathogenesis of human diseases. For future clinical applications, rather than intervention strategies to target general autophagy, the specific targeting of selective autophagy pathways may enhance the efficacy of therapeutic strategies. Further research into selective autophagy in the lung and other organs will allow for the development new therapeutic interventions.

\section{REFERENCES}

Campbell, G. R., and Spector, S. A. (2012). Vitamin D inhibits human immunodeficiency virus type 1 and Mycobacterium tuberculosis infection in macrophages through the induction of autophagy. PLoS Pathog. 8:e1002689. doi: 10.1371/journal.ppat.1002689

Chen, B. B., Coon, T. A., Glasser, J. R., Zou, C., Ellis, B., Das, T., et al. (2014a). E3 ligase subunit Fbxo15 and PINK1 kinase regulate cardiolipin synthase 1 stability and mitochondrial function in pneumonia. Cell Rep. 7, 476-487. doi: 10.1016/j.celrep.2014.02.048

Chen, G., Han, Z., Feng, D., Chen, Y., Chen, L., Wu, H., et al. (2014b). A regulatory signaling loop comprising the PGAM5 phosphatase and CK2 controls receptormediated mitophagy. Mol. Cell 54, 362-377. doi: 10.1016/j.molcel.2014.02.034

Chen, Z. H., Lam, H. C., Jin, Y., Kim, H. P., Cao, J., Lee, S. J., et al. (2010). Autophagy protein microtubule-associated protein 1 light chain-3B (LC3B) activates extrinsic apoptosis during cigarette smoke-induced emphysema. Proc. Natl. Acad. Sci. U.S.A. 107, 18880-18885. doi: 10.1073/pnas.1005574107

Choi, A. M., Ryter, S. W., and Levine, B. (2013). Autophagy in human health and disease. N. Engl. J. Med. 368, 1845-1846. doi: 10.1056/NEJMra1205406

Cloonan, S. M., Lam, H. C., Ryter, S. W., and Choi, A. M. (2014). "Ciliophagy": the consumption of cilia components by autophagy. Autophagy 10, 532-534. doi: 10.4161/auto.27641

Dal-Re, R. (2011). Worldwide behavioral research on major global causes of mortality. Health Educ. Behav. 38, 433-440. doi: 10.1177/1090198111402197

Dolinay, T., Kim, Y. S., Howrylak, J., Hunninghake, G. M., An, C. H., Fredenburgh, L., et al. (2012). Inflammasome-regulated cytokines are critical mediators of acute lung injury. Am. J. Respir. Crit. Care Med. 185, 1225-1234. doi: 10.1164/rccm.201201-0003OC

Frank, M., Duvezin-Caubet, S., Koob, S., Occhipinti, A., Jagasia, R., Petcherski, A., et al. (2012). Mitophagy is triggered by mild oxidative stress in a mitochondrial fission dependent manner. Biochim. Biophys. Acta 1823, 2297-2310. doi: 10.1016/j.bbamcr.2012.08.007

Fu, M., St-Pierre, P., Shankar, J., Wang, P. T., Joshi, B., and Nabi, I. R. (2013). Regulation of mitophagy by the Gp78 E3 ubiquitin ligase. Mol. Biol. Cell 24, 1153-1162. doi: 10.1091/mbc.E12-08-0607

Gegg, M. E., Cooper, J. M., Chau, K. Y., Rojo, M., Schapira, A. H., and Taanman, J. W. (2010). Mitofusin 1 and mitofusin 2 are ubiquitinated in a PINK1/parkindependent manner upon induction of mitophagy. Hum. Mol. Genet. 19, 48614870. doi: 10.1093/hmg/ddq419

Geisler, S., Holmstrom, K. M., Skujat, D., Fiesel, F. C., Rothfuss, O. C., Kahle, P. J., et al. (2010). PINK1/Parkin-mediated mitophagy is dependent on VDAC1 and p62/SQSTM1. Nat. Cell Biol. 12, 119-131. doi: 10.1038/ncb2012

Gomes, L. C., and Dikic, I. (2014). Autophagy in antimicrobial immunity. Mol. Cell 54, 224-233. doi: 10.1016/j.molcel.2014.03.009

Goncharova, E. A. (2013). mTOR and vascular remodeling in lung diseases: current challenges and therapeutic prospects. FASEB J. 27, 1796-1807. doi: 10.1096/fj.12222224

Hailey, D. W., Rambold, A. S., Satpute-Krishnan, P., Mitra, K., Sougrat, R., Kim, P. K., etal. (2010). Mitochondria supply membranes for autophagosome biogenesis during starvation. Cell 141, 656-667. doi: 10.1016/j.cell.2010. 04.009

Hamasaki, M., Furuta, N., Matsuda, A., Nezu, A., Yamamoto, A., Fujita, N., et al. (2013). Autophagosomes form at ER-mitochondria contact sites. Nature 495, 389-393. doi: 10.1038/nature11910

Intemann, C. D., Thye, T., Niemann, S., Browne, E. N., Amanua Chinbuah, M., Enimil, A., et al. (2009). Autophagy gene variant IRGM-261T contributes to 
protection from tuberculosis caused by Mycobacterium tuberculosis but not by $M$. africanum strains. PLoS Pathog. 5:e1000577. doi: 10.1371/journal.ppat.1000577

Itakura, E., Kishi-Itakura, C., and Mizushima, N. (2012). The hairpin-type tail-anchored SNARE syntaxin 17 targets to autophagosomes for fusion with endosomes/lysosomes. Cell 151, 1256-1269. doi: 10.1016/j.cell.2012.11.001

Kaufmann, A., Beier, V., Franquelim, H. G., and Wollert, T. (2014). Molecular mechanism of autophagic membrane-scaffold assembly and disassembly. Cell 156, 469-481. doi: 10.1016/j.cell.2013.12.022

Kirkin, V., McEwan, D. G., Novak, I., and Dikic, I. (2009). A role for ubiquitin in selective autophagy. Mol. Cell 34, 259-269. doi: 10.1016/j.molcel.2009.04.026

Klionsky, D. J., Cregg, J. M., Dunn, W. A. Jr., Emr, S. D., Sakai, Y., Sandoval, I. V. et al. (2003). A unified nomenclature for yeast autophagy-related genes. Dev. Cell 5, 539-545. doi: 10.1016/S1534-5807(03)00296-X

Krymskaya, V. P., Snow, J., Cesarone, G., Khavin, I., Goncharov, D. A., Lim, P. N., et al (2011). mTOR is required for pulmonary arterial vascular smooth muscle cell proliferation under chronic hypoxia. FASEB J. 25, 1922-1933. doi: 10.1096/fj.10175018

Lahm, T., and Petrache, I. (2012). LC3 as a potential therapeutic target in hypoxia-induced pulmonary hypertension. Autophagy 8, 1146-1147. doi: 10.4161/auto. 20520

Lam, H. C., Cloonan, S. M., Bhashyam, A. R., Haspel, J. A., Singh, A., Sathirapongsasuti, J. F., et al. (2013). Histone deacetylase 6-mediated selective autophagy regulates COPD-associated cilia dysfunction. J. Clin. Invest. 123, 5212-5230. doi: 10.1172/JCI69636

Lam, K. K., Zheng, X., Forestieri, R., Balgi, A. D., Nodwell, M., Vollett, S., et al. (2012) Nitazoxanide stimulates autophagy and inhibits mTORC1 signaling and intracellular proliferation of Mycobacterium tuberculosis. PLoS Pathog. 8:e1002691. doi: 10.1371/journal.ppat.1002691

Lee, S., Lee, S. J., Coronata, A. A., Fredenburgh, L. E., Chung, S. W., Perrella, M. A. et al. (2014). Carbon monoxide confers protection in sepsis by enhancing beclin 1-dependent autophagy and phagocytosis. Antioxid. Redox Signal. 20, 432-442. doi: 10.1089/ars.2013.5368

Lee, S. J., Smith, A., Guo, L., Alastalo, T. P., Li, M., Sawada, H., et al. (2011). Autophagic protein LC3B confers resistance against hypoxia-induced pulmonary hypertension. Am. J. Respir. Crit. Care Med. 183, 649-658. doi: 10.1164/rccm.201005-0746OC

Levine, B., and Kroemer, G. (2008). Autophagy in the pathogenesis of disease. Cell 132, 27-42. doi: 10.1016/j.cell.2007.12.018

Levine, B., Mizushima, N., and Virgin, H. W. (2011). Autophagy in immunity and inflammation. Nature 469, 323-335. doi: 10.1038/nature09782

Liang, X., Wei, S. Q., Lee, S. J., Fung, J. K., Zhang, M., Tanaka, A., et al. (2013) p62 sequestosome $1 /$ light chain $3 \mathrm{~b}$ complex confers cytoprotection on lung epithelial cells after hyperoxia. Am. J. Respir. Cell Mol. Biol. 48, 489-496. doi: $10.1165 / \mathrm{rcmb} .2012-0017 \mathrm{OC}$

Liang, X. H., Jackson, S., Seaman, M., Brown, K., Kempkes, B., Hibshoosh, H., et al. (1999). Induction of autophagy and inhibition of tumorigenesis by beclin 1. Nature 402, 672-676. doi: 10.1038/45257

Liu, L., Feng, D., Chen, G., Chen, M., Zheng, Q., Song, P., et al. (2012). Mitochondrial outer-membrane protein FUNDC1 mediates hypoxia-induced mitophagy in mammalian cells. Nat. Cell Biol. 14, 177-185. doi: 10.1038/ncb2422

Loewenberg, S. (2012). India reports cases of totally drug-resistant tuberculosis. Lancet 379:205. doi: 10.1016/S0140-6736(12)60085-3

Long, L., Yang, X., Southwood, M., Lu, J., Marciniak, S. J., Dunmore, B. J., et al. (2013). Chloroquine prevents progression of experimental pulmonary hypertension via inhibition of autophagy and lysosomal bone morphogenetic protein type II receptor degradation. Circ. Res. 112, 1159-1170. doi: 10.1161/CIRCRESAHA.111.300483

Mizumura, K., Cloonan, S. M., Nakahira, K., Bhashyam, A. R., Cervo, M., Kitada, T., et al. (2014). Mitophagy-dependent necroptosis contributes to the pathogenesis of COPD. J. Clin. Invest. 124, 3987-4003. doi: 10.1172/JCI74985

Mizushima, N., and Komatsu, M. (2011). Autophagy: renovation of cells and tissues. Cell 147, 728-741. doi: 10.1016/j.cell.2011.10.026

Mizushima, N., Yoshimori, T., and Levine, B. (2010). Methods in Mammalian autophagy research. Cell 140, 313-326. doi: 10.1016/j.cell.2010. 01.028

Monick, M. M., Powers, L. S., Walters, K., Lovan, N., Zhang, M., Gerke, A., et al (2010). Identification of an autophagy defect in smokers' alveolar macrophages. J. Immunol. 185, 5425-5435. doi: 10.4049/jimmunol.1001603
Nakahira, K., Haspel, J. A., Rathinam, V. A., Lee, S. J., Dolinay, T., Lam, H. C., et al. (2011). Autophagy proteins regulate innate immune responses by inhibiting the release of mitochondrial DNA mediated by the NALP3 inflammasome. Nat. Immunol. 12, 222-230. doi: 10.1038/ni.1980

Nakahira, K., Kyung, S. Y., Rogers, A. J., Gazourian, L., Youn, S., Massaro, A. F., et al. (2013). Circulating mitochondrial DNA in patients in the ICU as a marker of mortality: derivation and validation. PLoS Med. 10:e1001577; discussion e1001577. doi: 10.1371/journal.pmed.1001577

Nath, S., Dancourt, J., Shteyn, V., Puente, G., Fong, W. M., Nag, S., et al. (2014). Lipidation of the LC3/GABARAP family of autophagy proteins relies on a membrane-curvature-sensing domain in Atg3. Nat. Cell Biol. 16, 415-424. doi: $10.1038 /$ ncb2940

Niu, H., Yamaguchi, M., and Rikihisa, Y. (2008). Subversion of cellular autophagy by Anaplasma phagocytophilum. Cell. Microbiol. 10, 593-605. doi: 10.1111/j.14625822.2007.01068.x

Pampliega, O., Orhon, I., Patel, B., Sridhar, S., Diaz-Carretero, A., Beau, I., et al. (2013). Functional interaction between autophagy and ciliogenesis. Nature 502, 194-200. doi: 10.1038/nature12639

Ratner, V., Sosunov, S. A., Niatsetskaya, Z. V., Utkina-Sosunova, I. V., and Ten, V. S. (2013). Mechanical ventilation causes pulmonary mitochondrial dysfunction and delayed alveolarization in neonatal mice. Am. J. Respir. Cell Mol. Biol. 49, 943-950. doi: $10.1165 / \mathrm{rcmb} .2012-0172 \mathrm{OC}$

Ratner, V., Starkov, A., Matsiukevich, D., Polin, R. A., and Ten, V. S. (2009). Mitochondrial dysfunction contributes to alveolar developmental arrest in hyperoxia-exposed mice. Am. J. Respir. Cell Mol. Biol. 40, 511-518. doi: 10.1165/rcmb.2008-0341RC

Ravikumar, B., Moreau, K., Jahreiss, L., Puri, C., and Rubinsztein, D. C. (2010). Plasma membrane contributes to the formation of pre-autophagosomal structures. Nat. Cell Biol. 12, 747-757. doi: 10.1038/ncb2078

Redmann, M., Dodson, M., Boyer-Guittaut, M., Darley-Usmar, V., and Zhang, J. (2014). Mitophagy mechanisms and role in human diseases. Int. J. Biochem. Cell Biol. 53, 127-133. doi: 10.1016/j.biocel.2014.05.010

Sawa-Makarska, J., Abert, C., Romanov, J., Zens, B., Ibiricu, I., and Martens, S. (2014). Cargo binding to Atg19 unmasks additional Atg8 binding sites to mediate membrane-cargo apposition during selective autophagy. Nat. Cell Biol. 16, 425433. doi: $10.1038 /$ ncb 2935

Schiavi, A., and Ventura, N. (2014). The interplay between mitochondria and autophagy and its role in the aging process. Exp. Gerontol. 56, 147-153. doi: 10.1016/j.exger.2014.02.015

Schnaith, A., Kashkar, H., Leggio, S. A., Addicks, K., Kronke, M., and Krut, O. (2007). Staphylococcus aureus subvert autophagy for induction of caspaseindependent host cell death. J. Biol. Chem. 282, 2695-2706. doi: 10.1074/jbc. M609784200

Schroder, K., and Tschopp, J. (2010). The inflammasomes. Cell 140, 821-832. doi: 10.1016/j.cell.2010.01.040

Semenza, G. L. (2011). Oxygen sensing, homeostasis, and disease. N. Engl. J. Med. 365, 537-547. doi: 10.1056/NEJMra1011165

Sentelle, R. D., Senkal, C. E., Jiang, W., Ponnusamy, S., Gencer, S., Selvam, S. P., et al. (2012). Ceramide targets autophagosomes to mitochondria and induces lethal mitophagy. Nat. Chem. Biol. 8, 831-838. doi: 10.1038/nchembio.1059

Settembre, C., Di Malta, C., Polito, V. A., Garcia Arencibia, M., Vetrini, F., Erdin, S., et al. (2011). TFEB links autophagy to lysosomal biogenesis. Science 332, 1429-1433. doi: 10.1126/science.1204592

Shen, H. M., and Mizushima, N. (2014). At the end of the autophagic road: an emerging understanding of lysosomal functions in autophagy. Trends Biochem. Sci. 39, 61-71. doi: 10.1016/j.tibs.2013.12.001

Singh, S. B., Davis, A. S., Taylor, G. A., and Deretic, V. (2006). Human IRGM induces autophagy to eliminate intracellular mycobacteria. Science 313, 1438-1441. doi: 10.1126/science. 1129577

Stenmark, K. R., Fagan, K. A., and Frid, M. G. (2006). Hypoxia-induced pulmonary vascular remodeling: cellular and molecular mechanisms. Circ. Res. 99, 675-691. doi: 10.1161/01.RES.0000243584.45145.3f

Stolz, A., Ernst, A., and Dikic, I. (2014). Cargo recognition and trafficking in selective autophagy. Nat. Cell Biol. 16, 495-501. doi: 10.1038/ ncb2979

Sureshbabu, A., and Bhandari, V. (2013). Targeting mitochondrial dysfunction in lung diseases: emphasis on mitophagy. Front. Physiol. 4:384. doi: $10.3389 /$ fphys. 2013.00384 
Tanaka, A., Jin, Y., Lee, S. J., Zhang, M., Kim, H. P., Stolz, D. B., et al. (2012). Hyperoxia-induced LC3B interacts with the Fas apoptotic pathway in epithelial cell death. Am. J. Respir. Cell Mol. Biol. 46, 507-514. doi: 10.1165/rcmb.2009$0415 \mathrm{OC}$

Tang, Z., Lin, M. G., Stowe, T. R., Chen, S., Zhu, M., Stearns, T., et al. (2013). Autophagy promotes primary ciliogenesis by removing OFD1 from centriolar satellites. Nature 502, 254-257. doi: 10.1038/nature12606

Tooze, S. A., and Yoshimori, T. (2010). The origin of the autophagosomal membrane. Nat. Cell Biol. 12, 831-835. doi: 10.1038/ncb0910-831

Tsukada, M., and Ohsumi, Y. (1993). Isolation and characterization of autophagydefective mutants of Saccharomyces cerevisiae. FEBS Lett. 333, 169-174. doi 10.1016/0014-5793(93)80398-E

Udwadia, Z. F., Amale, R. A., Ajbani, K. K., and Rodrigues, C. (2012). Totally drug-resistant tuberculosis in India. Clin. Infect. Dis. 54, 579-581. doi: 10.1093/cid/cir889

Vergne, I., Chua, J., Singh, S. B., and Deretic, V. (2004). Cell biology of Mycobac terium tuberculosis phagosome. Annu. Rev. Cell Dev. Biol. 20, 367-394. doi 10.1146/annurev.cellbio.20.010403.114015

Vestbo, J., Hurd, S. S., Agusti, A. G., Jones, P. W., Vogelmeier, C., Anzueto A., etal. (2013). Global strategy for the diagnosis, management, and prevention of chronic obstructive pulmonary disease: GOLD executive summary. Am. J. Respir. Crit. Care Med. 187, 347-365. doi: 10.1164/rccm.2012040596PP

Wang, W., Liu, J., Ma, A., Miao, R., Jin, Y., Zhang, H., et al. (2014). mTORC1 Is Involved in hypoxia-induced pulmonary hypertension through the activation of Notch3. J. Cell. Physiol. 229, 2117-2125. doi: 10.1002/jcp. 24670

Wang, X., Winter, D., Ashrafi, G., Schlehe, J., Wong, Y. L., Selkoe, D., et al (2011). PINK1 and Parkin target Miro for phosphorylation and degradation to arrest mitochondrial motility. Cell 147, 893-906. doi: 10.1016/j.cell.2011. 10.018

Watson, R. O., Manzanillo, P. S., and Cox, J. S. (2012). Extracellular M. tuberculosis DNA targets bacteria for autophagy by activating the host DNA-sensing pathway. Cell 150, 803-815. doi: 10.1016/j.cell.2012.06.040

Waxman, A. B., and Kolliputi, N. (2009). IL-6 protects against hyperoxiainduced mitochondrial damage via Bcl-2-induced Bak interactions with mitofusins. Am. J. Respir. Cell Mol. Biol. 41, 385-396. doi: 10.1165/rcmb. 2008-0302OC

Wrighton, K. H. (2013). Cytoskeleton: autophagy and ciliogenesis come together. Nat. Rev. Mol. Cell Biol. 14:687. doi: 10.1038/nrm3686
Wu, H., Xue, D., Chen, G., Han, Z., Huang, L., Zhu, C., et al. (2014). The BCL2L1 and PGAM5 axis defines hypoxia-induced receptor-mediated mitophagy. Autophagy 10, 1712-1725. doi: 10.4161/auto. 29568

Yamamoto, A., and Simonsen, A. (2011). The elimination of accumulated and aggregated proteins: a role for aggrephagy in neurodegeneration. Neurobiol. Dis. 43, 17-28. doi: 10.1016/j.nbd.2010.08.015

Yan, L., Li, Y., Duan, H., Yang, H., Wu, J., Qian, P., et al. (2014). Regulator of calcineurin 1-1L protects cardiomyocytes against hypoxia-induced apoptosis via mitophagy. J. Cardiovasc. Pharmacol. 64, 310-317. doi: 10.1097/FJC.0000000000000121

Yano, T., and Kurata, S. (2011). Intracellular recognition of pathogens and autophagy as an innate immune host defence. J. Biochem. 150, 143-149. doi: $10.1093 / \mathrm{jb} / \mathrm{mvr} 083$

Youle, R. J., and Narendra, D. P. (2011). Mechanisms of mitophagy. Nat. Rev. Mol. Cell Biol. 12, 9-14. doi: 10.1038/nrm3028

Zhang, Y., Sauler, M., Shinn, A. S., Gong, H., Haslip, M., Shan, P., et al. (2014). Endothelial PINK1 mediates the protective effects of NLRP3 deficiency during lethal oxidant injury. J. Immunol. 192, 5296-5304. doi: 10.4049/jimmunol.1400653

Zhou, R., Yazdi, A. S., Menu, P., and Tschopp, J. (2011). A role for mitochondria in NLRP3 inflammasome activation. Nature 469, 221-225. doi: 10.1038 /nature09663

Conflict of Interest Statement: The authors declare that the research was conducted in the absence of any commercial or financial relationships that could be construed as a potential conflict of interest.

Received: 15 September 2014; accepted: 23 October 2014; published online: 05 November 2014.

Citation: Mizumura K, Choi AMK and Ryter SW (2014) Emerging role of selective autophagy in human diseases. Front. Pharmacol. 5:244. doi: 10.3389/fphar.2014.00244 This article was submitted to Inflammation Pharmacology, a section of the journal Frontiers in Pharmacology.

Copyright (c) 2014 Mizumura, Choi and Ryter. This is an open-access article distributed under the terms of the Creative Commons Attribution License (CC BY). The use, distribution or reproduction in other forums is permitted, provided the original author(s) or licensor are credited and that the original publication in this journal is cited, in accordance with accepted academic practice. No use, distribution or reproduction is permitted which does not comply with these terms. 